\title{
Robust Fault Tolerant Control for a Class of Time-Delay Systems with Multiple Disturbances
}

\author{
Songyin Cao ${ }^{1,2}$ and Jianzhong Qiao ${ }^{2}$ \\ ${ }^{1}$ Department of Automation, College of Information Engineering, Yangzhou University, Yangzhou 225127, China \\ ${ }^{2}$ National Key Laboratory on Aircraft Control Technology, School of Instrumentation Science and Opto-Electronics Engineering, \\ Beihang University, Beijing 100191, China
}

Correspondence should be addressed to Songyin Cao; sycao@yzu.edu.cn

Received 11 April 2013; Accepted 25 May 2013

Academic Editor: Yang Yi

Copyright (c) 2013 S. Cao and J. Qiao. This is an open access article distributed under the Creative Commons Attribution License, which permits unrestricted use, distribution, and reproduction in any medium, provided the original work is properly cited.

\begin{abstract}
A robust fault tolerant control (FTC) approach is addressed for a class of nonlinear systems with time delay, actuator faults, and multiple disturbances. The first part of the multiple disturbances is supposed to be an uncertain modeled disturbance and the second one represents a norm-bounded variable. First, a composite observer is designed to estimate the uncertain modeled disturbance and actuator fault simultaneously. Then, an FTC strategy consisting of disturbance observer based control (DOBC), fault accommodation, and a mixed $H_{2} / H_{\infty}$ controller is constructed to reconfigure the considered systems with disturbance rejection and attenuation performance. Finally, simulations for a flight control system are given to show the efficiency of the proposed approach.
\end{abstract}

\section{Introduction}

To reduce the influence of model uncertainties and system disturbances, there are several control approaches focusing on nonlinear systems with unknown disturbances (see the survey paper [1] and references therein). The methodologies can be mainly classified into disturbance attenuation methods (such as $\mathrm{H}_{\infty}$ and $\mathrm{H}_{2}$ control) and disturbance rejection approaches (such as output regulation theory, disturbance observer based control). The disturbance attenuation approaches have conservativeness for bounded stochastic disturbance. The disturbance rejection methods are established based on model-matching conditions. It has been shown that multiple disturbances exist in most practical systems. The idea of disturbance observer based control (DOBC) is to construct an observer to estimate and compensate some external disturbances [2]. A composite control scheme combining DOBC and PD (proportional derivative) control for flexible spacecraft attitude control was proposed in the presence of model uncertainty, elastic vibration, and external disturbances [3]. For nonlinear systems with multiple disturbances, it has been seen that the $H_{\infty}$ and variable structure control have been integrated with DOBC in $[4,5]$. In [6], a composite DOBC and adaptive control approach were proposed for a class of nonlinear systems with multiple disturbances. By constructing a disturbance compensation gain vector in the composite control law, a nonlinear robust $\mathrm{DOBC}$ was proposed to attenuate the mismatched disturbances and the influence of parameter variations from output channels [7].

In order to increase the reliability and safety of practical engineering, the issues of fault diagnosis and fault tolerant control (FTC) have become an attractive topic and have been paid much attention in recent years (see [8-13] and references therein). It is difficult to accommodate faults if the disturbances and faults exist simultaneously in the controlled systems. In [14], an optimal fault tolerant control approach was proposed for the nonlinear systems, where generalized $H_{\infty}$ optimization was applied to estimate the fault and attenuate the disturbances. In [15], a robust observer was proposed to simultaneously estimate system states, faults, and their finite time derivatives and attenuate disturbances; then an FTC approach was designed based on their estimations. For systems with modeled disturbance, a fault diagnosis 
approach based on disturbance observer was firstly proposed in [16] with disturbance rejection performance. For the nonlinear system with multiple disturbances, [17] addressed a fault tolerant control approach with disturbance rejection and attenuation performances. It is well known that time delay frequently occurs in many practical systems, such as manufacturing systems, telecommunication, and economic systems. Therefore, the problem of fault accommodation for time-delay systems has been a hot topic in the control field. Many important results have been reported in the literature (see [18-21] and references therein). In [18], an adaptive fuzzy fault accommodation control approach was proposed for nonlinear time-delay system. In [19], a fault accommodation was addressed for time-varying delay system using adaptive fault diagnosis observer. [20] dealt with fault tolerant guaranteed cost controller design problem for linear time-delay system against actuator faults.

In this paper, FTC problem is discussed for a class of time-delay systems with actuator fault and multiple disturbances. The first part of multiple disturbances is modeled disturbance formulated by an exogenous system and the second one is norm bounded uncertain variable. A composite observer is designed to estimate the modeled disturbance and time-varying fault. Then, an FTC scheme is addressed with disturbance rejection and attenuation performance by combining fault accommodation and DOBC with a robust $\mathrm{H}_{2} / \mathrm{H}_{\infty}$ controller.

\section{Model Description}

In this paper, we consider the following nonlinear system with time-varying faults, time-delay, and multiple disturbances simultaneously:

$$
\begin{gathered}
\dot{x}(t)=A x(t)+A_{d} x(t-\tau)+G g(x(t)) \\
+J[u(t)+F(t)]+J_{1} d_{1}(t)+J_{2} d_{2}(t) \\
y(t)=C x(t),
\end{gathered}
$$

where $x(t) \in R^{n}$ is system state, $u(t)$ is control input, and $y(t) \in R^{m}$ represents output variable. $F(t)$ is timevarying actuator fault to be diagnosed. $A, A_{d}, C, G, J, J_{1}$, and $J_{2}$ represent coefficient matrices of the system with suitable dimensions. $\tau$ is a known constant delay. The modeled external disturbance $d_{1}(t)$ is supposed to be generated by a linear exogenous system described by

$$
\begin{gathered}
\dot{\omega}(t)=W \omega(t)+J_{3} \delta(t), \\
d_{1}(t)=V \omega(t),
\end{gathered}
$$

where $\omega(t)$ is state variable, $W \in R^{p \times p}$, and $V$ and $J_{3}$ are known parameter matrices of the exogenous system. $\delta(t)$ is additional disturbance which results from perturbations and uncertainties in the exogenous system. The disturbances $d_{2}(t)$ and $\delta(t)$ are supposed to have the bounded $\mathrm{H}_{2}$ norm.
For a known matrix $U_{1}, g(x(t))$ is a known nonlinear vector function that is supposed to satisfy $g(0)=0$ and the following norm condition:

$$
\left\|g\left(x_{1}(t)\right)-g\left(x_{2}(t)\right)\right\| \leq\left\|U_{1}\left(x_{1}(t)-x_{2}(t)\right)\right\|
$$

for any $x_{1}(t)$ and $x_{2}(t)$.

The following assumptions are required so that the considered problem can be well-posed in this paper.

Assumption 1. $(A, J)$ is controllable; $\left(W, J_{1} V\right)$ is observable.

Assumption 2. $\operatorname{rank}\left(J, J_{1}\right)=\operatorname{rank}(J)$.

Remark 1. In practical engineering, the exogenous model (2) can represent many kinds of disturbances including harmonic disturbance signal caused by vibration, unknown constant load in the motor, inertial sensor drift represented by first-order Gaussian Markov process, and so on. Compared with the previous works $[17,19,20]$, both the time-delay and multiple disturbances are simultaneously considered in this paper. Furthermore, the modeled disturbance $d_{1}(t)$ and control input are assumed in different channels, while in [17] are in the same channel.

\section{Robust Fault Tolerant Controller Design}

3.1. Disturbance Observer. In order to reject the modeled external disturbance, disturbance observer should be designed in this subsection. In this paper, we only consider the case of available states. The disturbance observer is formulated as

$$
\begin{gathered}
\widehat{\omega}(t)=\xi(t)-L x(t), \\
\widehat{d}_{1}(t)=V \widehat{\omega}(t),
\end{gathered}
$$

where $\xi(t)$ is auxiliary variable generated by

$$
\begin{aligned}
\dot{\xi}(t)= & \left(W+L J_{1} V\right)[\xi(t)-L x(t)]+L \\
\times & {\left[A x(t)+A_{d} x(t-\tau)+G g(x(t))\right.} \\
& \left.+J u(t)+J u_{f c}(t)\right]
\end{aligned}
$$

$\widehat{\omega}(t)$ is estimation of $\omega(t), \widehat{d}_{1}(t)$ is estimation of modeled disturbance $d_{1}(t)$, matrix $L$ is the disturbance observer gain to be determined later, and $u_{f c}(t)=\widehat{F}(t)$ is compensation term to be designed in fault diagnosis observer, where $\widehat{F}(t)$ is denoted as an estimation of fault $F(t)$.

By defining $e_{\omega}(t)=\omega(t)-\widehat{\omega}(t)$ and $e_{F}(t)=F(t)-\widehat{F}(t)$, estimation error system can be obtained from (1), (2), (4), and (5) to show the following:

$$
\dot{e}_{\omega}(t)=\left(W+L J_{1} V\right) e_{\omega}(t)+L J e_{F}(t)+L J_{2} d_{2}(t)+J_{3} \delta(t) .
$$

In the following subsection, we will construct a fault diagnosis observer with disturbance estimation so that the modeled disturbance can be rejected and fault can be diagnosed. 
3.2. Fault Diagnosis Observer. The following fault diagnosis observer is constructed to diagnose the time-varying actuator fault:

$$
\begin{gathered}
\widehat{F}(t)=\eta(t)-K x(t), \\
\dot{\eta}(t)=K J[\eta(t)-K x(t)] \\
+K\left[A x(t)+A_{d} x(t-\tau)\right. \\
\left.+G g(x(t))+J u(t)+J_{1} u_{d c}(t)\right],
\end{gathered}
$$

where $\widehat{F}(t)$ is estimation of $F(t)$. The disturbance observer based control term $u_{d c}(t)=\widehat{d}_{1}(t)$ is applied to reject modeled disturbance $d_{1}(t)$ by its estimation from disturbance observer. $K$ is the fault diagnosis observer gain to be determined later.

The fault estimation error system yields

$$
\begin{aligned}
\dot{e}_{F}(t) & =\dot{F}(t)-\dot{\widehat{F}}(t) \\
& =\dot{F}(t)+K J e_{F}(t)+K J_{1} V e_{\omega}(t)+K J_{2} d_{2}(t) .
\end{aligned}
$$

In the next subsection, a composite fault tolerant controller should be determined for reconfiguring the systems with disturbance rejection and attenuation performance.

3.3. Composite Fault Tolerant Controller. In this section, the object is to construct a control approach to guarantee that the system (1) is stable in the presence (or absence) of faults and multiple disturbances simultaneously for the considered time-delay system. The structure of composite fault tolerant controller is formulated as

$$
u(t)=u_{s c}(t)-u_{f c}(t)-J^{*} J_{1} u_{d c}(t),
$$

where $u_{f_{c}}(t)=\widehat{F}(t), u_{d c}(t)=\widehat{d}_{1}(t)$, and $u_{s c}(t)=S x(t), S$ is the state feedback controller gain to be determined later. Substituting (9) into (1), it can be seen that

$$
\begin{aligned}
\dot{x}(t)= & (A+J S) x(t)+A_{d} x(t-\tau)+G g(x(t)) \\
& +J e_{F}(t)+J_{1} d_{1}(t)-J J^{*} J_{1} \widehat{d}_{1}(t)+J_{2} d_{2}(t) .
\end{aligned}
$$

From Assumption 2, it can be seen that the vector space spanned by the columns of $J_{1}$ is a subset of the space spanned by the column vectors of $J[19]$; that is, $\operatorname{span}\left(J_{1}\right) \subset \operatorname{span}(J)$, which is equivalent to the existence of $J$, such that

$$
J_{1}-J J^{*} J_{1}=0 \text {. }
$$

Then, it can be concluded that

$$
\begin{aligned}
\dot{x}(t)= & (A+J S) x(t)+A_{d} x(t-\tau)+G g(x(t)) \\
& +J e_{F}(t)+J_{1} V e_{\omega}(t)+J_{2} d_{2}(t) .
\end{aligned}
$$

Combing estimation error equations (6) and (8) with (12) yields

$$
\begin{gathered}
\dot{\bar{x}}(t)=\bar{A} \bar{x}(t)+\bar{A}_{d} \bar{x}(t-\tau)+\bar{G} g(\bar{x}(t))+\bar{J} \bar{d}(t), \\
z_{2}(t)=\bar{C}_{2} \bar{x}(t)+\bar{C}_{d 2} \bar{x}(t-\tau), \\
z_{\infty}(t)=\bar{C}_{\infty} \bar{x}(t)+\bar{C}_{d \infty} \bar{x}(t-\tau)+D \bar{d}(t),
\end{gathered}
$$

where

$$
\begin{gathered}
\bar{x}(t)=\left[\begin{array}{c}
x(t) \\
e_{\omega}(t) \\
e_{F}(t)
\end{array}\right], \quad \bar{A}=\left[\begin{array}{ccc}
A+J S & J_{1} V & J \\
0 & W+L J_{1} V & L J \\
0 & K J_{1} V & K J
\end{array}\right], \\
\bar{G}=\left[\begin{array}{c}
G \\
0 \\
0
\end{array}\right], \\
\bar{A}_{d}=\left[\begin{array}{ccc}
A_{d} & 0 & 0 \\
0 & 0 & 0 \\
0 & 0 & 0
\end{array}\right], \quad \bar{J}=\left[\begin{array}{ccc}
J_{2} & 0 & 0 \\
L J_{2} & J_{3} & 0 \\
K J_{2} & 0 & I
\end{array}\right], \\
\bar{d}(t)=\left[\begin{array}{l}
d_{2}(t) \\
\delta(t) \\
\dot{F}(t)
\end{array}\right], \\
g(\bar{x}(t))=g(x(t))
\end{gathered}
$$

$z_{2}(t)$ is $H_{2}$ reference output,

$$
z_{2}(t)=C_{21} x(t)+C_{22}\left[e_{\omega}^{T}(t) e_{F}^{T}(t)\right]^{T}+C_{d 2} x(t-\tau)
$$

$z_{\infty}(t)$ is $H_{\infty}$ reference output,

$$
\begin{aligned}
z_{\infty}(t)= & C_{\infty 1} x(t)+C_{\infty 2}\left[e_{\omega}^{T}(t) e_{F}^{T}(t)\right]^{T} \\
& +C_{d \infty} x(t-\tau)+D \bar{d}(t)
\end{aligned}
$$

where $C_{21}, C_{22}, C_{d 2}, C_{\infty 1}, C_{\infty 2}, C_{d \infty}$, and $D$ are the selected weighting matrices.

Definition 2. For constants $\gamma_{1}>0, \gamma_{2}>0$, and $\gamma_{3}>0$, the $H_{\infty}$ performance is denoted as follows:

$$
\begin{aligned}
J_{\infty}= & \left\|z_{\infty}(t)\right\|^{2}-\gamma_{1}^{2}\left\|d_{2}(t)\right\|^{2}-\gamma_{2}^{2}\|\delta(t)\|^{2} \\
& -\gamma_{3}^{2}\|\dot{F}(t)\|^{2}-\delta\left(P_{1}, Q\right),
\end{aligned}
$$

where

$$
\delta\left(P_{1}, Q\right)=\phi^{T}(0) P_{1} \phi(0)+\int_{-d}^{0} \phi^{T}(\tau) Q \phi(\tau) d \tau
$$

Definition 3. The $H_{2}$ performance measure for (13) is defined as $J_{2}=\left\|z_{2}(t)\right\|^{2}$. 
Remark 4. Compared with [17], $H_{2} / H_{\infty}$ mixed multiobjective optimization technique is used for the composite system (13). In the proposed approach, the modeled disturbance and fault are rejected by their estimations, while $H_{\infty}$ performance is adopted to attenuate norm bounded uncertain disturbances and $\mathrm{H}_{2}$ performance index is applied to optimize estimation error.

At this stage, the objective is to find $K, L$, and $S$ such that system (13) is stable. The following result provides a design method based on convex optimization technology [22].

Theorem 5. If for the parameter $\lambda>0, r_{i}(i=1, \ldots, 4)$, matrices $C_{21}, C_{22}, C_{d 2}, C_{\infty 1}, C_{\infty 2}, C_{d \infty}$, and $D$, there exist matrices $P_{0}>0, P_{2}>0, Q_{0}>0, R_{0}$, and $R_{2}$ and constants $\gamma_{1}>0, \gamma_{2}>0$, and $\gamma_{3}>0$, if the following LMI-based optimization problem holds:

$$
\min \left\{r_{1} \gamma_{1}+r_{2} \gamma_{2}+r_{3} \gamma_{3}+r_{4} \phi^{T}(0) P_{0}^{-1} \phi(0)\right\}
$$

subject to

$$
\left[\begin{array}{ccccccccc}
\Xi_{1} & A_{d} Q_{0} & G & \bar{V} & \Xi_{15} & P_{0} U_{1}^{T} & P_{0} C_{\infty 1}^{T} & P_{0} C_{21}^{T} & P_{0} \\
* & -Q_{0} & 0 & 0 & 0 & 0 & C_{d \infty}^{T} & C_{d 2}^{T} & 0 \\
* & * & -\frac{1}{\lambda} I & 0 & 0 & 0 & 0 & 0 & 0 \\
* & * & * & \Xi_{4} & \Xi_{45} & 0 & C_{\infty 2}^{T} & C_{22}^{T} & 0 \\
* & * & * & * & \Xi_{5} & 0 & D^{T} & 0 & 0 \\
* & * & * & * & * & -\lambda I & 0 & 0 & 0 \\
* & * & * & * & * & * & -I & 0 & 0 \\
* & * & * & * & * & * & * & -I & 0 \\
* & * & * & * & * & * & * & * & -Q_{0}
\end{array}\right]
$$

$<0$

where

$$
\begin{gathered}
\Xi_{1}=\operatorname{sym}\left(A P_{0}+J R_{0}\right), \quad \Xi_{15}=\left[\begin{array}{lll}
J_{2} & 0 & 0
\end{array}\right], \\
\Xi_{4}=\operatorname{sym}\left(P_{2} \bar{W}+R_{2} \bar{V}\right),
\end{gathered}
$$

$$
\begin{gathered}
\Xi_{45}=\left[\begin{array}{lll}
R_{2} J_{2} & P_{2} \bar{J}_{3} & P_{2} \bar{J}_{1}
\end{array}\right], \\
\Xi_{5}=\left[\begin{array}{ccc}
-\gamma_{1}^{2} I & 0 & 0 \\
0 & -\gamma_{2}^{2} I & 0 \\
0 & 0 & -\gamma_{3}^{2} I
\end{array}\right], \\
\bar{W}=\left[\begin{array}{cc}
W & 0 \\
0 & 0
\end{array}\right], \quad \bar{V}=\left[\begin{array}{ll}
J_{1} V & J
\end{array}\right], \\
\bar{J}_{1}=\left[\begin{array}{l}
0 \\
I
\end{array}\right], \quad \bar{J}_{3}=\left[\begin{array}{c}
J_{3} \\
0
\end{array}\right], \quad \bar{C}=\left[\begin{array}{ll}
C_{1} & C_{2}
\end{array}\right],
\end{gathered}
$$

then with gains $S=R_{0} P_{0}^{-1}$ and $\bar{L}=\left[\begin{array}{c}L \\ K\end{array}\right]=P_{2}^{-1} R_{2}$, error system (13) is stable and satisfies $J_{\infty}<0$ and $J_{2} \leq \delta\left(P_{1}, Q\right)$. The symmetric terms in a symmetric matrix are denoted by $*$. The symbol $\operatorname{sym}()$ represents $\operatorname{sym}(\Theta):=\Theta+\Theta^{T}$.

Proof. Consider the following Lyapunov function:

$$
\begin{aligned}
\Pi(t)= & x^{T}(t) P_{1} x(t)+\int_{t-\tau}^{t} x^{T}(\tau) Q x(\tau) d \tau+\bar{e}^{T}(t) P_{2} \bar{e}(t) \\
& +\lambda \int_{0}^{t}\left[\|U x(\tau)\|^{2}-\|g(\tau)\|^{2}\right] d \tau .
\end{aligned}
$$

It is verified that $\Pi(t) \geq 0$ holds for all arguments. Along with the trajectories of (13), it can be shown that

$$
\begin{aligned}
\dot{\Pi}(t)= & 2 x^{T}(t) P_{1} \dot{x}(t)+x^{T}(t) Q x(t)-x(t-\tau)^{T} Q x(t-\tau) \\
& +2 \bar{e}^{T}(t) P_{2} \dot{\bar{e}}(t)+\lambda\left[\|U x(t)\|^{2}-\|g(x(t))\|^{2}\right] \\
= & 2 x^{T}(t) P_{1}\left[(A+J S) x(t)+A_{d} x(t-\tau)+G g(x(t))\right. \\
& \left.\quad+J_{1} V e_{\omega}(t)+J e_{F}(t)+J_{2} d_{2}(t)\right] \\
& +x^{T}(t) Q x(t)-x(t-\tau)^{T} Q x(t-\tau) \\
& +\lambda x^{T}(t) U^{T} U x(t)-\lambda\|g(x(t))\|^{2}+2 \bar{e}^{T}(t) \\
& \times P_{2}\left[(\bar{W}+\bar{L} \bar{V}) \bar{e}(t)+\bar{L} J_{2} d_{2}(t)+\bar{J}_{3} \delta(t)+\bar{J}_{1} \dot{F}(t)\right] .
\end{aligned}
$$

In the absence of $\bar{d}(t)$ (i.e., $\bar{d}(t)=0$ ), it can be seen that

$$
\dot{V}(t)=s^{T}(t)\left[\Phi-\zeta \zeta^{T}\right] s(t),
$$

where

$$
s^{T}(t)=\left[\begin{array}{llll}
x^{T}(t) & x^{T}(t-\tau) & g^{T}(x(t)) & \bar{e}^{T}(t)
\end{array}\right],
$$$$
\zeta^{T}=\left[\begin{array}{llll}
C_{21} & C_{d 2} & 0 & C_{22}
\end{array}\right]
$$$$
\Phi=\left[\begin{array}{cccc}
\Phi_{11} & P_{1} A_{d}+C_{21}^{T} C_{d 2} & P_{1} G & P_{1} \bar{V}+C_{21}^{T} C_{22} \\
* & -Q+C_{d 2}^{T} C_{d 2} & 0 & 0 \\
* & * & -\lambda I & 0 \\
* & * & * & \Phi_{44}
\end{array}\right] \text {, }
$$ 


$$
\begin{gathered}
\Phi_{11}=\operatorname{sym}\left(P_{1}(A+J S)\right)+\lambda U^{T} U+Q+C_{21}^{T} C_{21}, \\
\Phi_{44}=\operatorname{sym}\left(P_{2}(W+\bar{L} \bar{V})\right)+C_{22}^{T} C_{22} .
\end{gathered}
$$

$$
\left[\begin{array}{ccccccc}
\Xi_{1}+P_{0} Q_{0}^{-1} P_{0}+\frac{1}{\lambda} P_{0} U_{0}^{T} U_{0} P_{0} & Q_{0} A_{d} & G & \bar{V} & \Xi_{15} & P_{0} C_{\infty 1}^{T} & P_{0} C_{21}^{T} \\
* & -Q_{0} & 0 & 0 & 0 & C_{d \infty}^{T} & C_{d 2}^{T} \\
* & * & -\frac{1}{\lambda} I & 0 & 0 & 0 & 0 \\
* & * & * & \Xi_{4} & \Xi_{45} & C_{\infty 2}^{T} & C_{22}^{T} \\
* & * & * & * & \Xi_{5} & 0 & 0 \\
* & * & * & * & * & -I & 0 \\
* & * & * & * & * & * & -I
\end{array}\right]<0 .
$$

From the first, second, third, fourth, and seventh columns and rows of the left matrix in inequality (26) it can be verified that

$$
\begin{aligned}
& \Phi_{0}= \\
& {\left[\begin{array}{ccccc}
\Xi_{1}+P_{0} Q_{0}^{-1} P_{0}+\frac{1}{\lambda} P_{0} U_{0}^{T} U_{0} P_{0} & Q_{0} A_{d} & G & \bar{V} & P_{0} C_{21}^{T} \\
* & -Q_{0} & 0 & 0 & C_{d 2}^{T} \\
* & * & -\frac{1}{\lambda} I & 0 & 0 \\
* & * & * & \Xi_{4} & C_{22}^{T} \\
* & * & * & * & -I
\end{array}\right]}
\end{aligned}
$$$$
<0 \text {. }
$$

Defining $P_{0}=P_{1}^{-1}, Q^{-1}=Q_{0}$, premultiplied and postmultiplied simultaneously by $\operatorname{diag}\left\{P_{0}^{-1}, Q_{0}^{-1}, I, I, I\right\}$, it can be seen by using Schur complement formula that $\Phi_{0}<0$ leads to $\Phi<0$. When $\Phi<0$ holds, we have

$$
\dot{V}(t)<-s^{T}(t) \zeta \zeta^{T} s(t)=-z_{2}^{T} z_{2} \leq 0
$$

It follows that (13) is asymptotically stable in the absence of the exogenous input $\bar{d}(t)$. Next, we consider the performances to be optimized for (13). Consider two auxiliary functions as follows:

$$
\begin{gathered}
J_{0}=z_{2}^{T}(t) z_{2}(t)+\dot{V}(t) \\
J_{1}=z_{\infty}^{T}(t) z_{\infty}(t)-\gamma_{1}^{2} d_{2}(t)^{T} d_{2}(t)-\gamma_{2}^{2} \delta(t)^{T} \delta(t) \\
-\gamma_{3}^{2} \dot{F}(t)^{T} \dot{F}(t)+\dot{V}(t)
\end{gathered}
$$

Following the definition of the $H_{2}$ performance, we only consider the case in the absence of $\bar{d}(t)$. It can be verified that $J_{0} \leq s^{T}(t) \Phi s(t)$.
In the presence of $\bar{d}(t)$, it can be seen that $J_{1}=q^{T}(t) \Psi q(t)$, where

$$
\begin{aligned}
& q^{T}(t)=\left[\begin{array}{lllll}
x^{T}(t) & x^{T}(t-\tau) & g^{T}(x(t)) & \bar{e}^{T}(t) & \bar{d}^{T}(t)
\end{array}\right], \\
& \Psi=\left[\begin{array}{ccccc}
\Psi_{11} & P_{1} A_{d} & P_{1} G & P_{1} \bar{V} & \Psi_{15} \\
* & -Q & 0 & 0 & 0 \\
* & * & -\frac{1}{\lambda} I & 0 & 0 \\
* & * & * & \Psi_{44} & \Psi_{45} \\
* & * & * & * & \Xi_{5}
\end{array}\right] \\
& +\left[\begin{array}{c}
C_{\infty 1}^{T} \\
C_{d \infty}^{T} \\
0 \\
C_{\infty 2}^{T} \\
0
\end{array}\right]\left[\begin{array}{c}
C_{\infty 1}^{T} \\
C_{d \infty}^{T} \\
0 \\
C_{\infty 2}^{T} \\
0
\end{array}\right]^{T},
\end{aligned}
$$

where

$$
\begin{gathered}
\Psi_{11}=\operatorname{sym}\left(P_{1} A+P_{1} J S\right)+\frac{1}{\lambda} U^{T} U+Q, \\
\Psi_{15}=\left[\begin{array}{lll}
P_{1} J_{2} & 0 & 0
\end{array}\right], \\
\Psi_{44}=\operatorname{sym}\left(P_{2} \bar{W}+P_{2} \bar{L} \bar{V}\right), \\
\Psi_{45}=\left[\begin{array}{lll}
P_{2} \bar{L} J_{2} & P_{2} \bar{J}_{3} & P_{2} \bar{J}_{1}
\end{array}\right] .
\end{gathered}
$$

Denote

$$
\Psi_{0}=\Psi+\left[\begin{array}{lllll}
C_{21} & C_{d 2} & 0 & C_{22} & 0
\end{array}\right]^{T}\left[\begin{array}{lllll}
C_{21} & C_{d 2} & 0 & C_{22} & 0
\end{array}\right] .
$$

It can be seen by using Schur complement formula that (20) leads to $\Psi_{0}<0$, and then $\Psi<0$ holds. It can be verified that both $J_{0}<0$ and $J_{1}<0$ hold. This completes the proof. 


\section{Simulation Examples}

In this section, we consider the longitudinal dynamics of $\mathrm{A} 4 \mathrm{D}$ aircraft at a flight condition of $15000 \mathrm{ft}$ altitude and 0.9 Mach given in [2]. The longitudinal dynamics can be denoted as

$$
\begin{aligned}
\dot{x}(t)= & A x(t)+G g(x(t))+J(u(t)+F(t)) \\
& +J_{1} d_{1}(t)+J_{2} d_{2}(t),
\end{aligned}
$$

where $x(t)$ are measurable by using sensors technique. The coefficient matrices of aircraft model are given by

$$
\begin{gathered}
A=\left[\begin{array}{cccc}
-0.0605 & 32.37 & 0 & 32.2 \\
-0.00014 & -1.475 & 1 & 0 \\
-0.0111 & -34.72 & -2.793 & 0 \\
0 & 0 & 1 & 0
\end{array}\right], \\
J=\left[\begin{array}{c}
0 \\
-0.1064 \\
-33.8 \\
0
\end{array}\right], \quad J_{1}=\left[\begin{array}{c}
0 \\
-0.0532 \\
-16.9000 \\
0
\end{array}\right], \quad J_{2}=\left[\begin{array}{c}
0.1 \\
0 \\
-3 \\
0.1
\end{array}\right] .
\end{gathered}
$$

It is supposed that

$$
G=\left[\begin{array}{llll}
0 & 0 & 50 & 0
\end{array}\right]^{T}, \quad g(x(t))=\sin (2 \pi 5 t) x_{2}(t) .
$$

then, the matrix $U$ can be selected as $U=\operatorname{diag}\{0,1,0,0\}$ and the norm condition (3) can be satisfied. $A_{d}=0.5 \times$ $A, \tau=2$. Periodic disturbance $d_{1}(t)$ caused by rotating aerial propeller is assumed to be an unknown harmonic disturbance described by (2) with

$$
W=\left[\begin{array}{cc}
0 & 5 \\
-5 & 0
\end{array}\right], \quad E=\left[\begin{array}{ll}
25 & 0
\end{array}\right], \quad H_{3}=\left[\begin{array}{l}
0.1 \\
0.1
\end{array}\right],
$$

$\delta(t)$ is the additional disturbance signal resulting from the perturbations and uncertainties in the exogenous system (2) and satisfies 2-norm boundedness. In simulation, we select $\delta(t)$ as the random signal with upper 2-norm bound 1 . Wind gust and system noises $d_{2}(t)$ can also be considered as the random signal with upper 2 -norm bounded.

The initial values of the states are supposed to be $x^{T}(0)=$ $\left[\begin{array}{llll}2 & -2 & 3 & 2\end{array}\right]$. For the reference output, it is denoted that

$$
\begin{gathered}
C_{\infty 1}=\left[\begin{array}{llll}
1 & 1 & 1 & 1
\end{array}\right], \quad C_{\infty 2}=\left[\begin{array}{lll}
1 & 1 & 1
\end{array}\right], \\
C_{d \infty}=\left[\begin{array}{llll}
0.1 & 0.1 & 0.1 & 0.1
\end{array}\right], \\
C_{21}=\left[\begin{array}{llll}
0.1 & 0.1 & 0.1 & 0.1
\end{array}\right], \quad C_{22}=\left[\begin{array}{llll}
0.1 & 0.1 & 0.1
\end{array}\right], \\
C_{d 2}=\left[\begin{array}{llll}
0.01 & 0.01 & 0.01 & 0.01
\end{array}\right] .
\end{gathered}
$$

For $\lambda=1, \gamma_{1}=1, \gamma_{2}=1$, and $\gamma_{3}=1$, it can be solved via LMI related to (20) that the gain of fault diagnosis observer (7) is

$$
K=\left[\begin{array}{llll}
20.9299 & 10.0231 & 1.3553 & 20.9682
\end{array}\right],
$$

the gain of disturbance observer (4) is

$$
L=\left[\begin{array}{cccc}
-0.2584 & -0.5402 & -0.0142 & -0.2610 \\
2.3246 & 0.7523 & 0.1526 & 2.3269
\end{array}\right],
$$

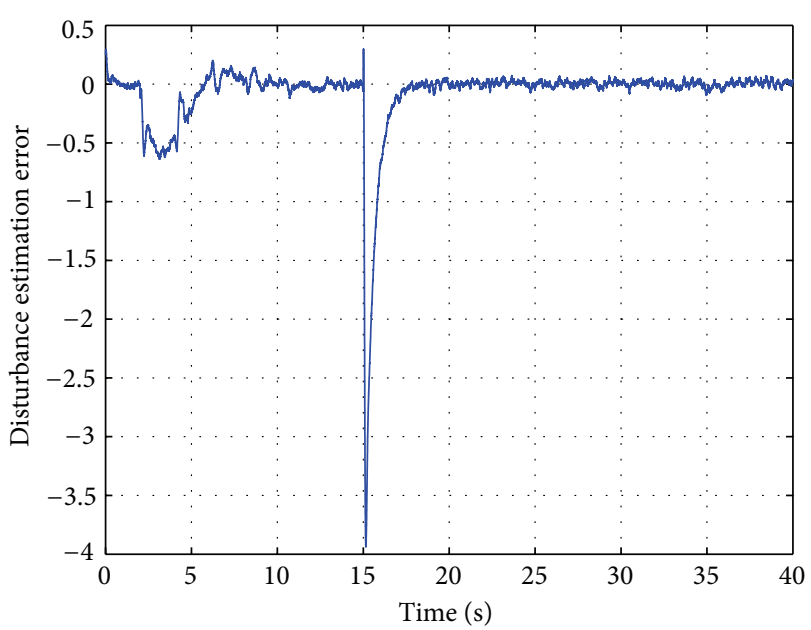

FIGURE 1: Disturbances estimation error in disturbance observer.

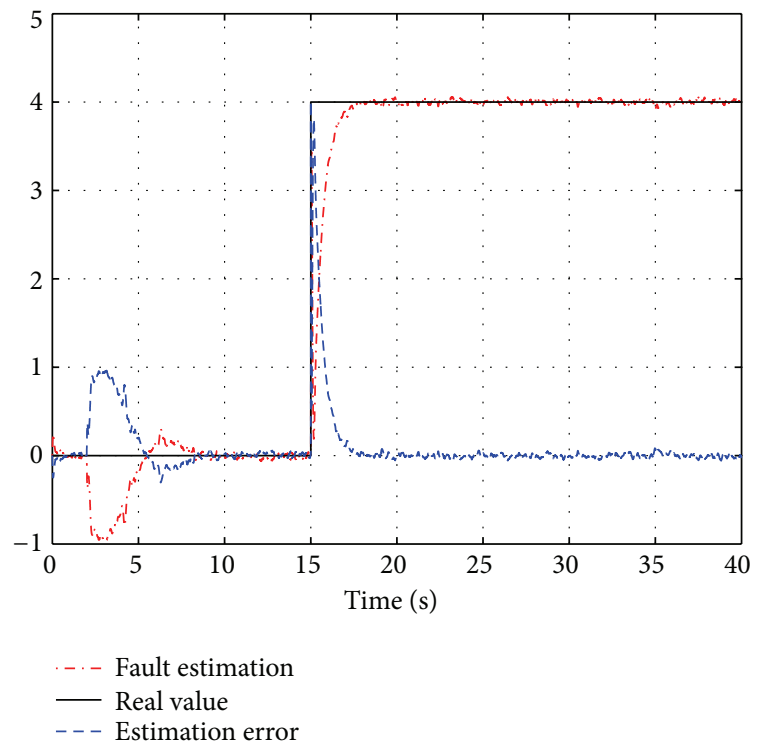

FIGURE 2: Bias fault, its estimation, and error.

and the gain of state feedback controller is

$$
S=\left[\begin{array}{llll}
89.6396 & 471.0670 & 74.2419 & 465.7941
\end{array}\right] .
$$

When the disturbance observer is constructed based on (4) and (5), the estimation error of exogenous disturbances is shown in Figure 1. The actuator bias fault is supposed to occur at 15 th second as $F=4$. The estimation and its error of fault with system disturbances are demonstrated in Figure 2, where the solid line represents the real fault signal, the dash-dotted line is its estimation, and the dash line denotes its estimation error. In Figure 3, the state response signals of the control system are illustrated. It can be seen that the proposed fault tolerant controller has a good control ability for configuring fault and rejecting and attenuating disturbances simultaneously. In Figure 4, the ramp fault is assumed to occur at 15 th second with slope 0.1 . From 


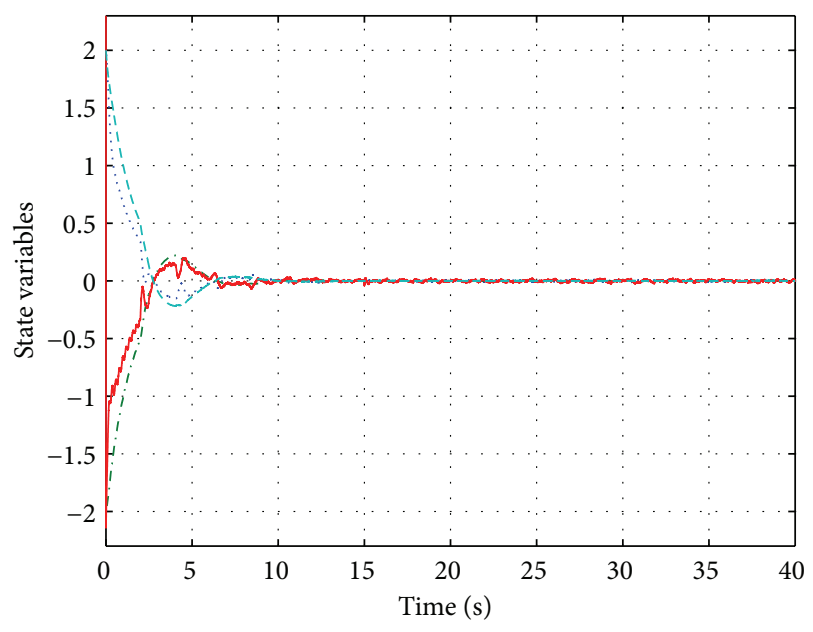

FIgURE 3: The responses of state variable.

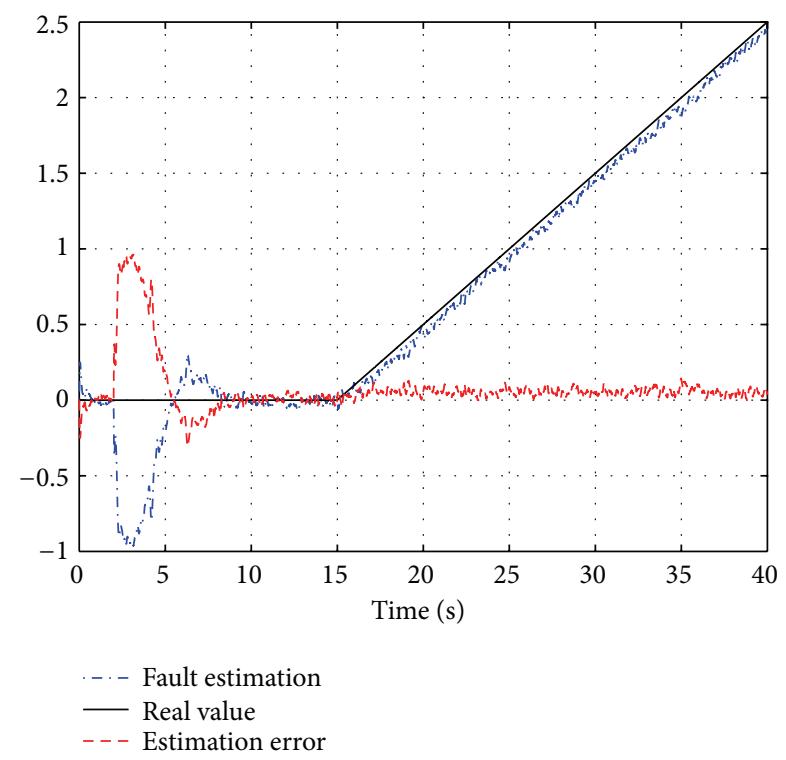

FIGURE 4: Ramp fault, its estimation, and error.

Figure 4, it is shown that the time-varying fault can also be well estimated.

\section{Conclusion}

In this paper, a robust antidisturbance fault tolerant control problem is investigated for nonlinear time delay systems with faults and multiple disturbances. There are the following features of the proposed algorithm compared with the previous results. First, the multiple disturbances and state timedelay are considered simultaneously in this paper. Second, an FTC scheme is addressed with disturbance rejection and attenuation performance by combining fault accommodation and DOBC with a mixed $H_{2} / H_{\infty}$ controller, with which the fault can be accommodated and the disturbances can be rejected and attenuated simultaneously. Finally, simulation for a flight control system is given to show the efficiency of the proposed approach.

\section{Acknowledgment}

This work is partially supported by the National Natural Science Foundation of China (Grant no. 61203195), Universities Natural Science Foundation of Jiangsu Province (Grant no. 12KJB510033), and China Postdoctoral Science Foundation (Grant no. 2013M530508).

\section{References}

[1] L. Guo, "Composite Hierarchical Anti-Disturbance Control (CHADC) for systems with multiple disturbances: survey and overview," in Proceedings of the 30th Chinese Control Conference (CCC '11), pp. 6193-6198, Yantai, China, July 2011.

[2] L. Guo and W.-H. Chen, "Disturbance attenuation and rejection for systems with nonlinearity via DOBC approach," International Journal of Robust and Nonlinear Control, vol. 15, no. 3, pp. 109-125, 2005.

[3] H. Liu, L. Guo, and Y. Zhang, "An anti-disturbance PD control scheme for attitude control and stabilization of flexible spacecrafts," Nonlinear Dynamics, vol. 67, no. 3, pp. 2081-2088, 2012.

[4] X. Wei and L. Guo, "Composite disturbance-observer-based control and $H_{\infty}$ control for complex continuous models," International Journal of Robust and Nonlinear Control, vol. 20, no. 1, pp. 106-118, 2010.

[5] M. Chen and W.-H. Chen, "Sliding mode control for a class of uncertain nonlinear system based on disturbance observer," International Journal of Adaptive Control and Signal Processing, vol. 24, no. 1, pp. 51-64, 2010.

[6] L. Guo and X. Wen, "Hierarchical anti-disturbance adaptive control for non-linear systems with composite disturbances and applications to missile systems," Transactions of the Institute of Measurement and Control, vol. 33, no. 8, pp. 942-956, 2011.

[7] J. Yang, W.-H. Chen, and S. Li, "Non-linear disturbance observer-based robust control for systems with mismatched disturbances/uncertainties," IET Control Theory and Applications, vol. 5, no. 18, pp. 2053-2062, 2011.

[8] M. Basseville and I. Nikiforov, "Fault isolation for diagnosis: nuisance rejection and multiple hypothesis testing," Annual Reviews in Control, vol. 26, pp. 189-202, 2002.

[9] J. J. Gertler, Fault Detection and Diagnosis in Engineering Systems, Marcel Dekker, New York, NY, USA, 1998.

[10] R. Isermann and P. Balle, "Trends in the application of model based fault detection and diagnosis of technical process," in Proceedings of the 13th World Congress, International Federation of Automatic Control (IFAC '96), pp. 1-12, San Francisco, Calif, USA, June 1996.

[11] R. J. Patton and J. Chen, Control and Dynamic Systems: Robust Fault Detection and Isolation (FDI) Systems, Academic, London, UK, 1996.

[12] M. M. Polycarpou and A. B. Trunov, "Learning approach to nonlinear fault diagnosis: detectability analysis," IEEE Transactions on Automatic Control, vol. 45, no. 4, pp. 806-812, 2000.

[13] Y. Zhang and J. Jiang, "Bibliographical review on reconfigurable fault-tolerant control systems," Annual Reviews in Control, vol. 32, no. 2, pp. 229-252, 2008.

[14] Y. Zhang, L. Guo, and H. Wang, "Robust filtering for fault tolerant control using output PDFs of non-Gaussian systems," 
IET Control Theory and Applications, vol. 1, no. 3, pp. 636-645, 2007.

[15] Z. Gao and S. X. Ding, "Actuator fault robust estimation and fault-tolerant control for a class of nonlinear descriptor systems," Automatica, vol. 43, no. 5, pp. 912-920, 2007.

[16] S. Cao and L. Guo, "Fault diagnosis with disturbance rejection performance based on disturbance observer," in Proceedings of the 48th IEEE Conference on Decision and Control held jointly with 28th Chinese Control Conference (CDC/CCC '09), pp. 6947-6951, Shanghai, China, December 2009.

[17] S. Cao, L. Guo, and X. Wen, "Fault tolerant control with disturbance rejection and attenuation performance for systems with multiple disturbances," Asian Journal of Control, vol. 13, no. 6, pp. 1056-1064, 2011.

[18] H. Wu, X. Qiang, and L. Guo, " $L_{\infty}$-gain adaptive fuzzy fault accommodation control design for nonlinear time-delay systems," IEEE Transactions on Systems, Man, and Cybernetics B, vol. 41, no. 3, pp. 817-827, 2011.

[19] B. Jiang, K. Zhang, and P. Shi, "Less conservative criteria for fault accommodation of time-varying delay systems using adaptive fault diagnosis observer," International Journal of Adaptive Control and Signal Processing, vol. 24, no. 4, pp. 322-334, 2010.

[20] D. Ye and G. H. Yang, "Adaptive fault-tolerant dynamic output feedback control for a class of linear time-delay systems," International Journal of Control, Automation, and Systems, vol. 6, no. 2, pp. 149-159, 2008.

[21] M. Zhong and Q.-L. Han, "Fault-tolerant master-slave synchronization for Lur'e systems using time-delay feedback control," IEEE Transactions on Circuits and Systems I, vol. 56, no. 7, pp. 1391-1404, 2009.

[22] L. Guo, F. Yang, and J. Fang, "Multiobjective filtering for nonlinear time-delay systems with nonzero initial conditions based on convex optimization," Circuits, Systems, and Signal Processing, vol. 25, no. 5, pp. 591-607, 2006. 


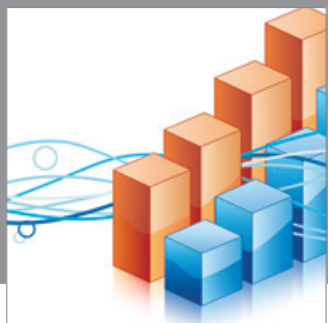

Advances in

Operations Research

mansans

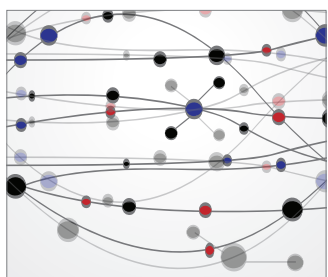

The Scientific World Journal
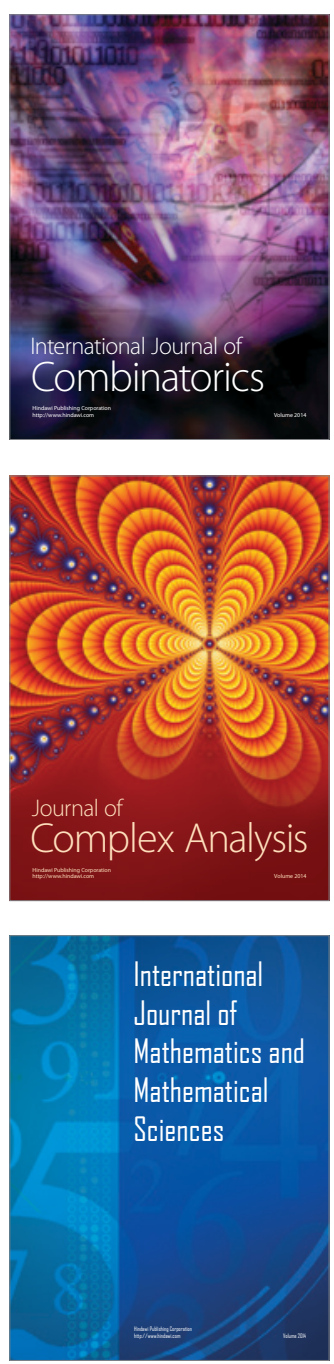
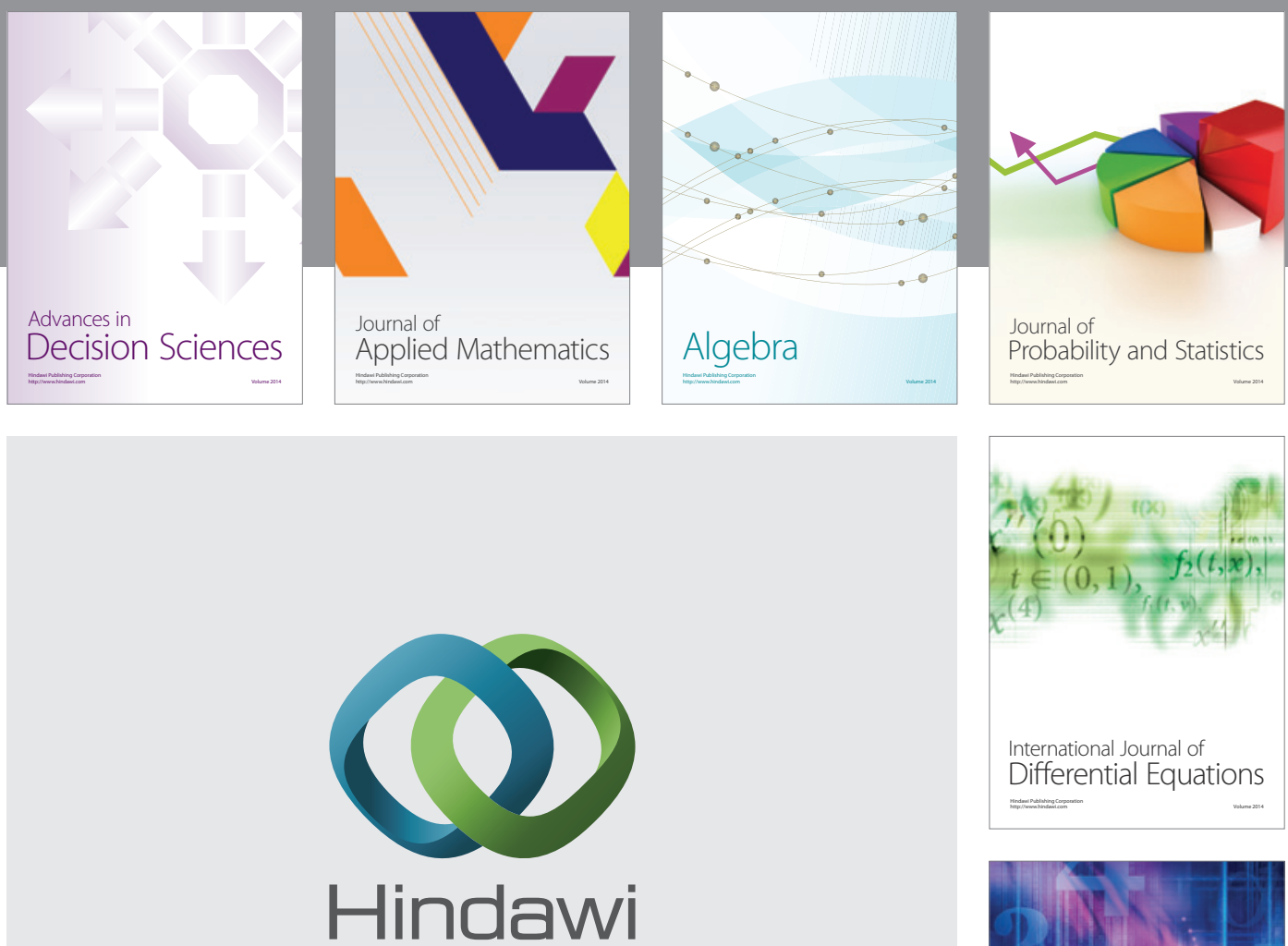

Submit your manuscripts at http://www.hindawi.com
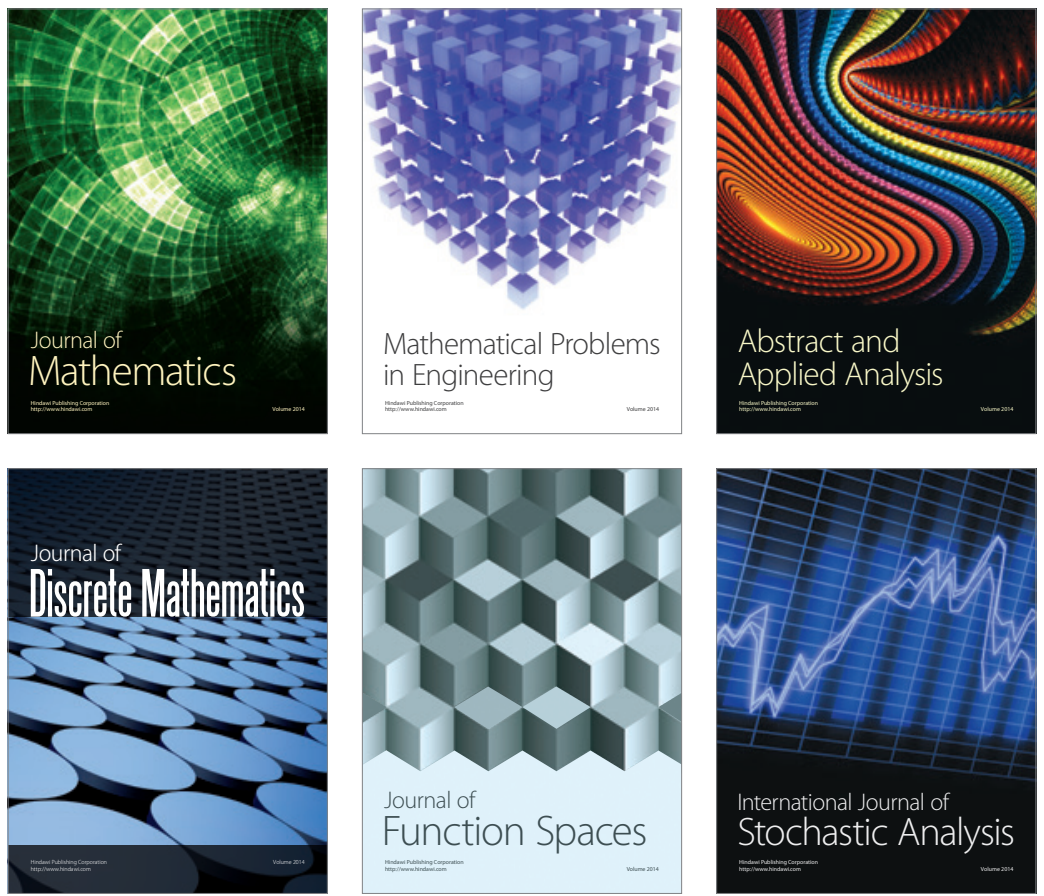

Journal of

Function Spaces

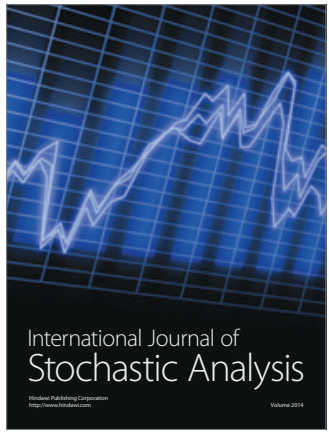

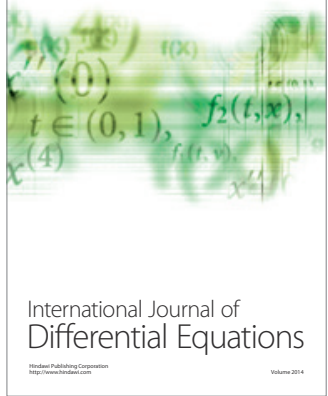
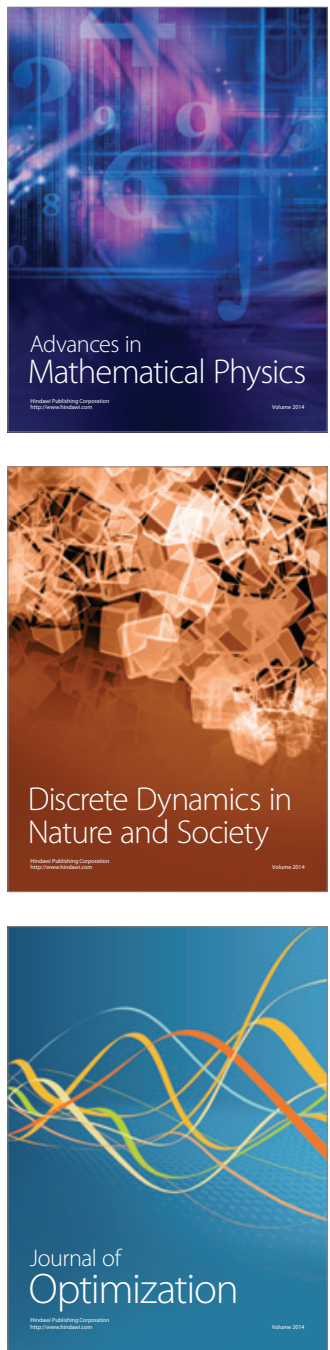